\title{
Effect of levocarnitine/iron saccharate combination on renal anaemia and oxidative stress in patients undergoing haemodialysis
}

\author{
Hong-Xia Cui* and En-Liang Wu \\ Hemodialysis Center, Binzhou People's Hospital, Binzhou, 256600, PR China
}

*For correspondence: Email: hxiacui@163.com

Received: 10 May 2016

Revised accepted: 10 September 2016

\begin{abstract}
Purpose: To investigate the effect of a combination of levocarnitine and iron saccharate on the treatment of renal anaemia and oxidative stress in patients undergoing haemodialysis.

Methods: A total of 156 patients with renal anaemia were divided randomly into control (78 cases) and test groups (78 cases). Patients in the control group were treated with erythropoietin (EPO), iron saccharate, and conventional symptomatic treatment, while patients in the test group were treated with levocarnitine additionally. Anaemia indices, oxidative stress indices, response rate, and EPO dose were compared.

Results: At week 28, the levels of hemoglobin b (Hb), hematocrit (Hct), serum ferritin (SF), and transferrin saturation (TSAT) in the test group were $92.72 \pm 12.51 \mathrm{~g} / \mathrm{L}, 34.74 \pm 5.89 \mathrm{vol} \%, 245.61 \pm$ $81.35 \mathrm{ng} / \mathrm{mL}$, and $24.34 \pm 5.32 \%$, respectively, which were much lower than the levels in the test group $(114.36 \pm 12.27 \mathrm{~g} / \mathrm{L}, 40.23 \pm 5.78 \mathrm{vol} \%, 345.07 \pm 85.93 \mathrm{ng} / \mathrm{mL}$, and $29.76 \pm 5.41 \%$, respectively; $p<$ $0.05)$. The levels of advanced oxidation protein products (AOPP) and malonaldehyde (MDA) in the test group were much higher than those of the control group (121.77 $\pm 31.65 \mathrm{nmol} / \mathrm{L}$ vs $89.65 \pm 30.53$ $\mathrm{nmol} / \mathrm{L} ; 9.58 \pm 2.64 \mathrm{nmol} / \mathrm{L}$ vs $4.81 \pm 2.57 \mathrm{nmol} / \mathrm{L}$, respectively $(p<0.05)$. EPO was maintained at a high dose from the beginning of treatment to week 28 in the control group, whereas in the test group, EPO dose was reduced gradually. The response rate in the test group was higher than that in the control group (92.30\% vs $76.90 \%$; $p<0.05)$.

Conclusion: Levocarnitine/iron saccharate combination had a significant positive effect on the treatment of renal anaemia. It effectively relieved oxidative stress reactions and reduced the dose of EPO required.
\end{abstract}

Keywords: Haemodialysis, Renal anaemia, Oxidative stress reaction, Levocarnitine, Iron saccharate, Erythropoietin

Tropical Journal of Pharmaceutical Research is indexed by Science Citation Index (SciSearch), Scopus, International Pharmaceutical Abstract, Chemical Abstracts, Embase, Index Copernicus, EBSCO, African Index Medicus, JournalSeek, Journal Citation Reports/Science Edition, Directory of Open Access Journals (DOAJ), African Journal Online, Bioline International, Open-J-Gate and Pharmacy Abstracts

\section{INTRODUCTION}

Renal anaemia, a commonly observed complication in patients with chronic renal failure during the terminal stage, occurs when the glomerular filtration rate (GFR) is reduced by over $50 \%$ [1,2]. Renal anaemia can induce abnormalities in multiple physiological functions, as well as cardiovascular and cerebrovascular diseases, thereby influencing the quality of life of patients with chronic kidney disease and lowering survival rates. Fortunately, erythropoietin (EPO) can relieve anaemic symptom in patients with chronic renal failure [3]. However, some patients with renal anaemia who undergo haemodialysis treatment respond poorly 
or not at all to EPO because of iron deficiency, chronic infection, and malnutrition [4,5]. Thus, supplementing with iron saccharate is usually regarded as an adjuvant therapy. However, researchers $[6,7]$ have found that iron saccharate can induce an increase in oxidative stress reactions and the occurrence of cardiovascular and cerebrovascular events. Thus, antioxidant interventions are also important in patients undergoing haemodialysis.

Levocarnitine, also called L-carnitine [8], is an amino acid that is extensively distributed in body tissues and is used for the treatment of several chronic renal failure - associated complications in patients who undergo long-term haemodialysis and develop secondary carnitine deficiency. Levocarnitine can significantly relieve malnutrition and antioxidation, allowing the EPO dose to be lowered, thus reducing the economic stress on patients. This study evaluate the curative effect of iron saccharate combined with levocarnitine in renal anaemia and oxidative stress. To this end, the clinical effects of iron saccharate alone and iron saccharate in combination with levocarnitine were compared in treating chronic renal anaemia in patients undergoing haemodialysis were compared.

\section{METHODS}

\section{Study setting, design and patient characteristics}

A total of 156 patients with renal anaemia who underwent haemodialysis between March 2011 and June 2014 at Binzhou People's Hospital, Shandong, China, were selected randomly. The study was approved by the Medical Ethics Committee of Binzhou People's Hospital (approval number: CHX20150518BZ), and conformed to the principles of the Declaration of Helsinki [9]. Those with stable disease conditions for at least 1 month, who underwent haemodialysis 2 or 3 times per week, who had a haemoglobin $(\mathrm{Hb})$ level $<90 \mathrm{~g} / \mathrm{L}$, a serum ferritin (SF) level $<200 \mathrm{ng} / \mathrm{mL}$, or a transferrin saturation (TSAT) $<20 \%$, and who signed the informed consent form (themselves or a family member) were included. Those who suffered from other severe complications or endocrine diseases or who had been treated with chalybeate were excluded.

The patients were divided into control $(n=78)$ and test $(n=78)$ groups using the random number table method. In the test group, there were 40 males and 38 females, with ages ranging from 34 to 66 years (average $49.46 \pm$
10.13) and a haemodialysis period of $17.6-43.3$ months. In the control group, there were 42 males and 36 females, with ages ranging from 35 to 67 years (average $49.57 \pm 9.97$ ) and a haemodialysis period of 18.2 - 43.6 months. Age, gender, and duration of haemodialysis were not significantly different between the groups $(p>$ $0.05)$; thus, the results were comparable.

\section{Treatment}

Based on maintenance haemodialysis, patients in the control group were treated with EPO and iron saccharate. EPO (Chengdu Diao Jiuhong Pharmaceutical Co., Ltd., Sichuang, China; batch No.: S20020060) at doses $150-200 \mathrm{U} / \mathrm{kg}$ was injected subcutaneously. $\mathrm{Hb}$ levels were measured every 4 weeks. If the $\mathrm{Hb}$ level increased to $<10 \mathrm{~g} / \mathrm{L}$, then the EPO dose was increased by $25 \mathrm{U} / \mathrm{kg}$ each time; if the $\mathrm{Hb}$ level exceeded the normal value, then the EPO dose was decreased by $25 \mathrm{U} / \mathrm{kg}$ each time. Moreover, $100 \mathrm{mg}$ iron saccharate (Nanjing Hengsheng Pharmaceutical Co., Ltd., Jiangsu, China; batch no. H20113004) were injected through the vein end of the dialysis tube after each haemodialysis session. In the treatment group, the same procedure as that in the control group was followed, but the patients were additionally injected intravenously with $1.0 \mathrm{~g}$ levocarnitine (batch no. H20050443; Haiyue Pharmaceutical Co., Ltd, Jilin, China) and $20 \mathrm{~mL}$ normal saline after haemodialysis. Patients in both groups were treated for 28 weeks.

\section{Determination of parameters}

Levels of $\mathrm{Hb}$, haematocrit (Hct), TSAT, advanced oxidation protein products (AOPP), and malonaldehyde (MDA) before treatment and after 28 weeks of treatment were compared. The maintenance doses of EPO in the groups were recorded at the beginning of treatment and at weeks 14 and 28. The curative effect was determined according to the changes in the anaemia and oxidative indices [10]. The treatment was considered significantly effective if the $\mathrm{Hb}$ level improved to at least $30 \mathrm{~g} / \mathrm{L}$, the level of Hct increased to at least $0.1 \mathrm{vol} \%$, or $\mathrm{Hb}$ and Hct both recovered to normal levels, and the anaemia symptoms improved significantly during treatment. The treatment was considered effective if the level of $\mathrm{Hb}$ improved to at least 15 $\mathrm{g} / \mathrm{L}$ or the Hct increased to at least $0.05 \mathrm{vol} \%$ and the anaemia symptoms were relieved by the end of treatment. The treatment was considered ineffective if the levels of $\mathrm{Hb}$ or Hct showed little to no change or the anaemia symptoms were aggravated. The formula for the overall response rate $(R)$ is as in Eq 1. 
$R(\%)=\{(S+C) / T\} 100$

$\mathrm{S}$ is the number of significantly effective cases, $\mathrm{C}$ the number of effective cases and $T$ the total number of cases.

\section{Statistical analysis}

The SPSS software (ver. 19.0) was used for statistical analysis. Data expressed as mean \pm standard deviation (SD), were analysed using the Student's test. Numerical data were compared using the $X^{2}$ test. $P$ values $<0.05$ were considered to indicate statistically significant differences.

\section{RESULTS}

\section{Haematological and oxidative stress indices}

Levels of Hb, Hct, SF, TSAT, AOPP, and MDA in the two groups showed no statistically significant difference before treatment (all $p>0.05$ ). After 28 weeks of treatment, the levels of $\mathrm{Hb}$, Hct, SF, and TSAT were significantly higher in the test group than the control group $(p<0.05)$, whereas the levels of AOPP and MDA were lower in the test group than in the control group $(p<0.05$; Table 1).

\section{Comparison of EPO doses between the two groups}

Throughout the treatment period, the required EPO dose in the control group remained relatively high, whereas the EPO dose in the test group was reduced gradually. At week 28 , the EPO dose in the test group was significantly lower than that in the control group (Table 2).

\section{Comparison of the curative effect between the two groups}

The results suggested that the overall response rate in the test group was significantly higher than that in the control group $24 \mathrm{~h}$ after treatment $(p<0.05$; Table 3$)$.

Table 1: Comparison of haematological and oxidative stress indices between the two groups (mean \pm SD)

\begin{tabular}{lccccc}
\hline Parameter & Time & Control group & Test group & t & $P$-value \\
\hline \multirow{2}{*}{$\mathrm{Hb}(\mathrm{g} / \mathrm{L})$} & Before & $74.87 \pm 14.30$ & $75.33 \pm 14.88$ & 0.128 & 0.899 \\
& After & $92.72 \pm 12.51$ & $114.36 \pm 12.27$ & 7.766 & 0.000 \\
$\mathrm{Hct}(\mathrm{vol} \%)$ & Before & $21.93 \pm 4.32$ & $21.42 \pm 4.50$ & 0.484 & 0.629 \\
& After & $34.74 \pm 5.89$ & $40.23 \pm 5.78$ & 4.185 & 0.000 \\
$\mathrm{SF}(\mathrm{ng} / \mathrm{mL})$ & Before & $118.37 \pm 58.37$ & $120.43 \pm 60.11$ & 0.157 & 0.876 \\
& After & $245.61 \pm 81.35$ & $345.07 \pm 85.93$ & 5.280 & 0.000 \\
$\mathrm{TSAT}(\%)$ & Before & $17.24 \pm 3.92$ & $17.33 \pm 3.93$ & 0.091 & 0.928 \\
& After & $24.34 \pm 5.32$ & $29.76 \pm 5.41$ & 4.480 & 0.000 \\
$\mathrm{AOPP}(\mathrm{nmol} / \mathrm{L})$ & Before & $82.62 \pm 22.47$ & $81.77 \pm 20.50$ & 0.174 & 0.863 \\
& After & $121.77 \pm 31.65$ & $89.65 \pm 30.53$ & 4.588 & 0.000 \\
$\mathrm{MDA}(\mathrm{nmol} / \mathrm{L})$ & Before & $4.23 \pm 1.53$ & $4.25 \pm 1.54$ & 0.058 & 0.954 \\
& After & $9.58 \pm 2.64$ & $4.81 \pm 2.57$ & 8.069 & 0.000 \\
\hline
\end{tabular}

Hb: haemoglobin B; Hct: hematocrit; SF: serum ferritin; TSAT: transferrin saturation; AOPP: advanced oxidation protein products; MDA: malonaldehyde; $t$ : Student's test; $P$ : the appearance probability of specimen results or more extreme results when original hypothesis is true

Table 2: Comparison of the erythropoietin dose between the two groups during treatment (mean $\pm \mathrm{SD}, \mathrm{U} /(\mathrm{kg} \cdot \mathrm{w})\}$

\begin{tabular}{lccc}
\hline Group & Baseline & Week 14 & Week 28 \\
\hline Control & $185.70 \pm 14.90$ & $179.30 \pm 12.60$ & $160.20 \pm 12.30$ \\
Treatment & $183.40 \pm 15.60$ & $163.10 \pm 11.70$ & $142.10 \pm 12.50$ \\
$t$ & 0.614 & 5.720 & 6.501 \\
$P$-value & 0.541 & 0.000 & 0.000 \\
\hline
\end{tabular}

Table 3: Comparison of the response rate between the two groups $(n)$

\begin{tabular}{lcccc}
\hline Group & Significantly effective & Effective & Ineffective & Response rate (\%) \\
& & & & \\
\hline Control & 24 & 36 & 22 & 76.92 \\
Treatment & 38 & 34 & 6 & $92.30^{*}$ \\
\hline
\end{tabular}

Note: * $P<0.05$, vs. the control group 


\section{DISCUSSION}

Renal anaemia is typically induced by chronic renal failure, caused by multiple organic renal diseases. The internal and external secretory functions of the kidney weaken or fail completely when chronic renal failure occurs. The pathogenesis of renal anaemia is complex. Its clinical manifestations are often masked by primary renal diseases, especially in the initial stages.

With the extensive application of renal dialysis, patients with chronic renal failure now tend to have longer survival periods; however, the number of cases of chronic renal anaemia is also increasing [11]. A study [12] suggested that $26.50 \%$ and $95.80 \%$ of 110 patients with chronic renal failure developed anaemia during the renal insufficiency period and the uraemia period, respectively. Most patients undergoing maintenance haemodialysis will likely develop chronic renal anaemia, and anaemia can severely influence treatment efficacy and quality of life. Therefore, there is an urgent need to treat chronic renal anaemia in patients undergoing maintenance haemodialysis.

According to a previous study [13], EPO deficiency is the leading cause of chronic renal anaemia. Consequently, treating renal anaemia with EPO has been promoted in the early stages of the disease, and EPO can correct anaemia symptoms in most patients. However, recent clinical investigations suggest that about $10 \%$ of patients with chronic renal anaemia show a poor response to EPO.

A clinical study [14] showed that levels of $\mathrm{Hb}$ and Hct may fail to reach normal values after treatment with EPO. The main reason is that iron deficiency greatly influences the curative effect of EPO treatment. Indeed, half of haemodialysis patients undergoing EPO treatment experience a poor curative effect due to iron deficiency. Repeated blood collection, residual blood in the dialyzer or pipeline, and the application of highdose EPO can all result in iron deficiency. Moreover, iron in the storage pool may not be released in a timely manner, leading to a functional iron deficiency and a microinflammatory state [15]. As a result, chalybeate is usually given to renal anaemia patients undergoing haemodialysis during EPO treatment.

Iron saccharate is an important drug used for iron supplementation. Sucrose molecules that surround $\mathrm{Fe}(\mathrm{OH})_{3}$, the core of iron saccharate, prevent its elimination by the kidney. However, studies [16,17] have suggested that iron saccharate can result in increased oxidative stress reactions, although it is considered safe and has a definite effect in treating renal anaemia. Oxidative stress refers to the release of a large number of oxygen radicals, caused by respiratory burst, which occurs when reduced coenzyme II on the membrane of leukocytes is activated.

Levocarnitine, also called L-carnitine, is an amino acid that is extensively distributed in the body tissues and is necessary for the metabolism of fatty acids [18]. It has been shown that levocarnitine can promote the synthesis of albumins, significantly improve the stability of the erythrocyte membrane, increase Hct, and transport long-chain fatty acids into the body [19]. Patients undergoing maintenance haemodialysis tend to have insufficient levocarnitine, due to a lack of levocarnitine before treatment, severe loss during treatment, and the consumption of levocarnitine during treatment. Levocarnitine deficiency can affect metabolism, increase the fragility of normal red blood cells, and shorten the life of these cells [20].

This study found that the levels of two major oxidative stress markers, AOPP and MDA, were increased significantly in both groups before treatment, suggesting the presence of oxidative stress. After 28 weeks of treatment, the levels of AOPP and MDA increased markedly in both groups, indicating that iron saccharate resulted in a further increase in oxidative stress. However, the increases in the levels of AOPP and MDA were less in the test group than the control group at week $28(p<0.05)$, suggesting that levocarnitine had an antioxidant effect that could relieve the oxidative stress induced by iron saccharate.

It was also found that the levels of $\mathrm{Hb}, \mathrm{Hct}, \mathrm{SF}$, and TAST of the patients were higher in the test group than the control group at week 28. The dose of EPO in the test group, which was decreased gradually, was lower than that in the control group at week 28 . This suggested that levocarnitine could improve the curative effect of EPO, and that the combined use could significantly relieve the anaemia symptoms of patients undergoing haemodialysis and lower the dose of EPO. The mechanism of action might be because levocarnitine promotes the absorption of EPO by acting on erythroid progenitor cells [21].

\section{Study limitations}

No adverse reaction was observed in the research subjects, which differs from the findings 
of other studies. It is considered that this was associated with the small sample size. Therefore, a study with a larger sample size is required to evaluate the safety of levocarnitine in combination with iron saccharate in treating renal anaemia.

\section{CONCLUSION}

Levocarnitine combined with iron saccharate has a positive therapeutic effect on renal anaemia in patients undergoing haemodialysis. The therapy significantly improves anaemia and oxidative stress indices and patient quality of life and reduces the damage caused by oxidative stress. However, the indications and conditions for administering the drugs must be established first, particularly in elderly patients whose physical and organ functions are weaker.

\section{DECLARATIONS}

\section{Acknowledgement}

The authors sincerely thank all who supported this work.

\section{Conflict of Interest}

No conflict of interest associated with this work.

\section{Contribution of Authors}

The authors declare that this work was done by the authors named in this article and all liabilities pertaining to claims relating to the content of this article will be borne by them.

\section{REFERENCES}

1. Xu ZZ, Zhu ZZ, Qi G, Chen YZ, Mou Y, Zhang R, Li J, Guo $Q R$. Clinical analysis of intravenous iron sucrose in the treatment of anemia of renal failure. Sichuan Med $J$ 2008; 29(8): 982-983.

2. Feng JH, Cui AD, Pu HM. Effects of Hematodialysis and Hematodiafiltration on Chronic Renal Failure Uremia in Patients with Anemia. Appl J Gen Pract 2011; 9(10): 1525-1526.

3. Zeng YR, Tan FJ. Observation of curative effect of iron sucrose in the treatment of anemia of patients undergoing hematodialysis. Chin Comm Doctors 2011; 13(268): 71-72.

4. Wang WR, Li QF. 12 cases of hematodialysis patients with secondary polycythemia. J Pract Med 2012; 28(23): 3963-3964.

5. Fu ZX. Curative effect of erythopietin, iron sucrose in combination with levocarnitine in the treatment of renal anemia of patients undergoing hematodialysis. Mod Med Health 2013; 29(15): 2350-2351.

6. Dimitrijevic ZM, Cvetkovic TP, Djordjevic VM, Pavlovic $D D$, Stefanovic NZ, Stojanovic IR, Paunovic GJ, Velickovic-Radovanovic RM. How the duration period of erythropoietin treatment influences the oxidative status of hemodialysis patients. Int J Med Sci 2012; 9(9): 808815.

7. Beguin $Y$, Jaspers A. Iron sucrose - characteristics, efficacy and regulatory aspects of an established treatment of iron deficiency and iron-deficiency anemia in a broad range of therapeutic areas. Expert Opin Pharmacother 2014; 15(14): 2087-2103.

8. Qin Y, Li Y. Observation of curative effect of levocarnitine in combination with erythopietin in the treatment of renal anemia of elder patients undergoing maintenance hemodialysis. Chin Med 2013; 8(3): 413.

9. Declaration of Helsinki. The 59th World Medical Association, 2008.

10. Wei F. Clinical study of levocarnitine in improving cardiac functions of patients undergoing maintenance hematodialysis. Contemp Med 2012; 18(36): 2-3.

11. Malaguarnera $M$, Vicari E, Calogero A, Cammalleri L, Di Fazio I, Gargante MP, Pennisi G, Risino C, Ranno S, Rampello $L$. Sexual dysfunction in chronic hepatitis $C$ virus patients treated with interferon alpha and ribavirin. J Interferon Cytokine Res 2008; 28(10): 603-609.

12. Wei $X H$. Observe the effect of levocarnitine combined with recombinant human erythropoietin treatment of renal anemia. Guangxi Med 2013; (9):1218-1219.

13. Saluk-Juszczak J, Olas B, Wachowicz B, Glowacki R, Bald E. L-carnitine modulates blood platelet oxidative stress. Cell Biol Toxicol 2010; 26: 355-365.

14. Wanic-Kossowska M, Kazmierski M. Combined therapy with $L$-carnitine and erythropoietin of anemia in chronic kidney failure patients undergoing hemodialysis. Pol Arch Med Wewn 2009; 117(1/2): 14-19.

15. El-Nakib GA, Mostafa T, Abbas TM, El-Shishtawy MM, Mabrouk MM, Sobh MA. Role of alpha-lipoic acid in the management of anemia in patients with chronic renal failure undergoing hemodialysis. Int J Nephrol Renovasc Dis 2013; 6: 161-168.

16. Prats $M$, Font $R$, García C, Muñoz-Cortés $M$, Cabré $C$, Jariod M, Romeu M, Giralt M, Martinez-Vea A. Oxidative stress markers in predicting response to treatment with ferric carboxymaltose in nondialysis chronic kidney disease patients. Clin Nephrol, 2014, 81(6): 419-426.

17. Hao LZ, Liu DL. The application of levocarnitine in the treatment of renal anemia and its effect on oxidative stress reaction. Shandong Med J 2011; 51(52): 112113.

18. Lu QH, Ding $G H$, Shi $M$, Li YM, Zhu L. Effects of levocarnitine on oxidative stress state of patients undergoing hemodialysis. Pract Clin Med 2014; 15(2): 32-34.

19. Zhou CX, Sun SS, Liu XF, Zhang ZX, Jiang $X F$. Observation of curative effect of iron sucrose injection in combination with levocarnitine in the treatment of renal 
Cui \& Wu

anemia of patients undergoing maintenance hemodialysis. Chin Pract Med 2013; 36(2): 182-183.

20. Liu JT, Wang YX. Effects of $L$ - carnitine with iron sucrose on anemia in patients undergoing hemodialysis. Clin Med 2009; 29(12): 19-20.
21. Jia $D Q$, Cao $Y$, Wang $C B$. Observation of curative effect of levocarnitine combined with EPO in treating uremic anemia. Shandong Med J, 2009; 49(9): 85-86. 